\title{
MODEL EXPERIMENT AND FIELD TEST OF PW-OWC TYPE WAVE POWER EXTRACTING BREAKWATER
}

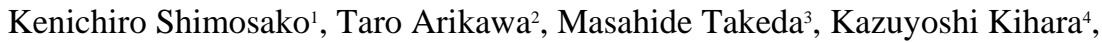 \\ Yasushi Hosokawa ${ }^{5}$, Takayuki Fueki ${ }^{6}$, Koichi Masuda ${ }^{7}$, Tomoki Ikoma ${ }^{8}$, \\ Shuichi Nagata ${ }^{9}$, Hiroki Osawa ${ }^{10}$, and Tsuyoshi Miyazaki ${ }^{11}$
}

\begin{abstract}
Many research studies on wave power conversion have been conducted in Japan over the past 30 years, but the outgrowth of these studies have yet to be put to practical use. In this study, medium-scale and large-scale hydraulic model experiments as well as prototype field test on a new oscillating water column with a projecting wall (PWOWC) wave power extracting breakwater were carried out in order to investigate the efficiency of energy conversion. The primary energy conversion efficiency of the PW-OWC converter is about $10 \%$ larger than that of the standard OWC type used in the medium-scale experiments. The primary energy conversion efficiency of the large scale experiments is about $20-30-\%$ smaller than that of the medium scale experiments. The secondary energy conversion efficiency ranges from 0.20 to 0.42 and becomes small when the wave period is short and the wave height is large.
\end{abstract}

Keywords: wave power extracting breakwater, model experiment, energy conversion efficiency

\section{INTRODUCTION}

Many research studies on wave power conversion have been conducted in Japan over the past 30 years (e.g. Takahashi et al.). However, the gains of these studies have yet to be put into practical use because of the high cost of power generation. The recently-developed oscillating water column (OWC) converter is constructed with a projecting wall (PW) in order to improve the efficiency of energy conversion (Ikoma et al.). The concept of the PW-OWC wave power extracting breakwater is shown in Fig. 1.

In this research, medium-scale and large-scale hydraulic model experiments on the PW-OWC wave extracting breakwater were carried out with the focus of attention on energy conversion efficiency. Turbine output power was also examined in the large scale experiments.

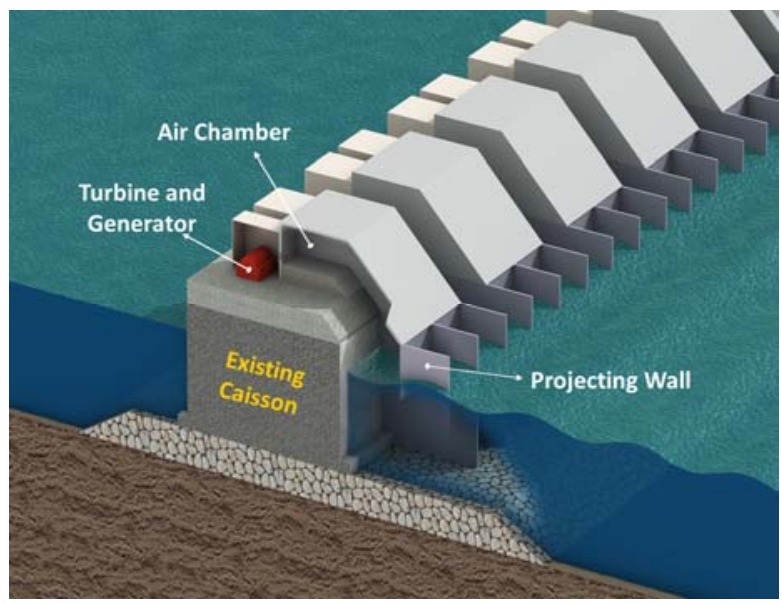

Figure 1. Concept of the PW-OWC wave power extracting breakwater.

\footnotetext{
${ }^{1}$ Port and Airport Research Institute, 3-1-1, Nagase, Yokosuka, Kanagawa, 239-0826 Japan

${ }^{2}$ Faculty of Science and Engineering, Chuo University, 1-13-27, Kasuga, Bunkyo-ku, Tokyo, 112-8551, Japan

${ }^{3}$ Research and Development Center, TOA Corporation, 3-7-1, Nishi-shinjuku, Shinjuku-ku, Tokyo, 163-1031, Japan

${ }^{4}$ MM Bridge Co., Ltd, 9-19, Nihonbashitomisawa-cho, Chuo-ku, Tokyo, 103-0006, Japan

${ }^{5}$ MM Bridge Co., Ltd, 9-19, Nihonbashitomisawa-cho, Chuo-ku, Tokyo, 103-0006, Japan

${ }^{6}$ Honma Corporation, 4, Kandaiwamoto-cho, Chiyoda-ku, Tokyo, 101-0033, Japan

${ }^{7}$ Nihon University, 7-24-1, Narashinodai, Funabashi, Chiba, 274-8501, Japan

${ }^{8}$ Nihon University, 7-24-1, Narashinodai, Funabashi, Chiba, 274-8501, Japan

${ }^{9}$ Saga University, 1, Honjo-machi, Saga, Saga, 840-8502, Japan

${ }^{10}$ Japan Agency for Marine-Earth Science and Technology, 2-15, Natsushima-cho, Yokosuka, Kanagawa, 237-0061 Japan

${ }^{11}$ Japan Agency for Marine-Earth Science and Technology, 2-15, Natsushima-cho, Yokosuka, Kanagawa, 237-0061, Japan
} 


\section{MEDIUM-SCALE EXPERIMENTS}

\section{Experimental setup}

Medium scale model experiments were carried out in the wave flume (105 m long, $2 \mathrm{~m}$ high, 0.8 m wide), reproducing the breakwater in Sakata Port with a 1/26 scale model. Figure 2 shows the cross section of the wave power extracting breakwater in medium scale experiment. The caisson was made of acrylic plate (Fig. 3), and it was installed in the flume (Fig. 4).

Pressures acting on the caisson, projecting walls and in the air chamber were measured using the small-sized pressure transducers as shown in Fig. 5. Wave gauges were installed in the air chamber to measure the up-and-down motion of the water level, as well as in front of the caisson to measure the wave height.

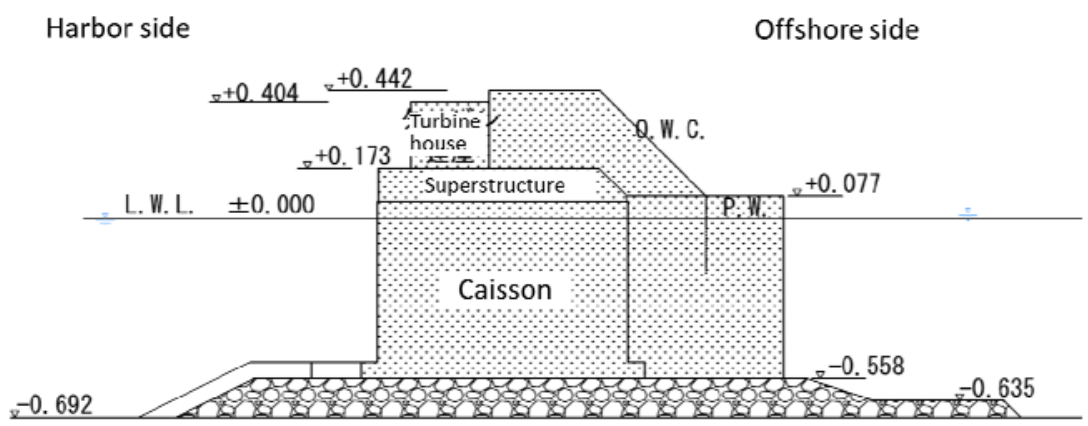

Figure 2. Cross-section of the wave power extracting breakwater in the medium-scale experiments.

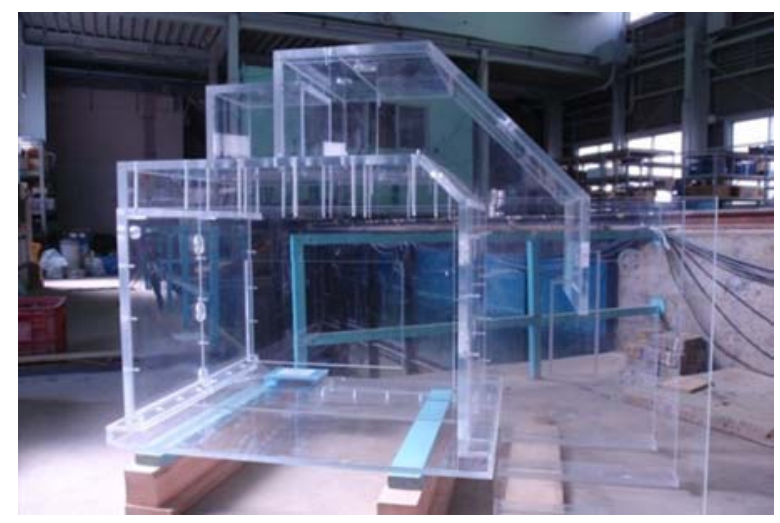

Figure 3. Acrylic model caisson.

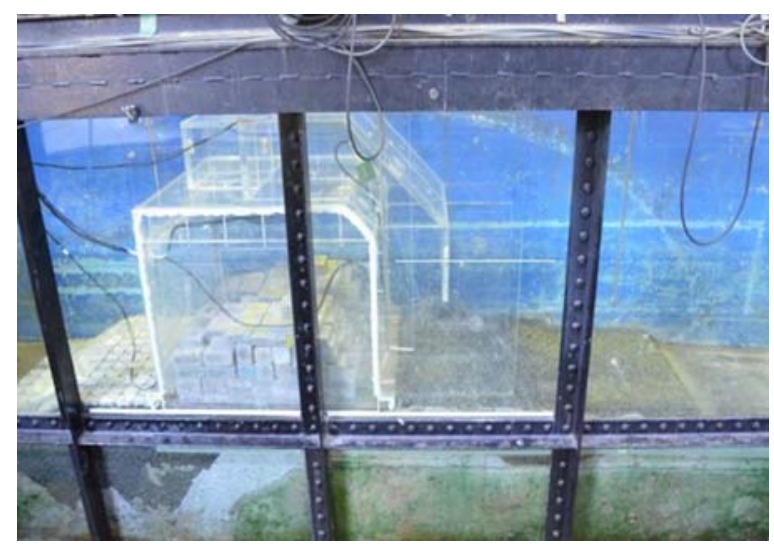

Figure 4. Model caisson installed in the wave flume. 


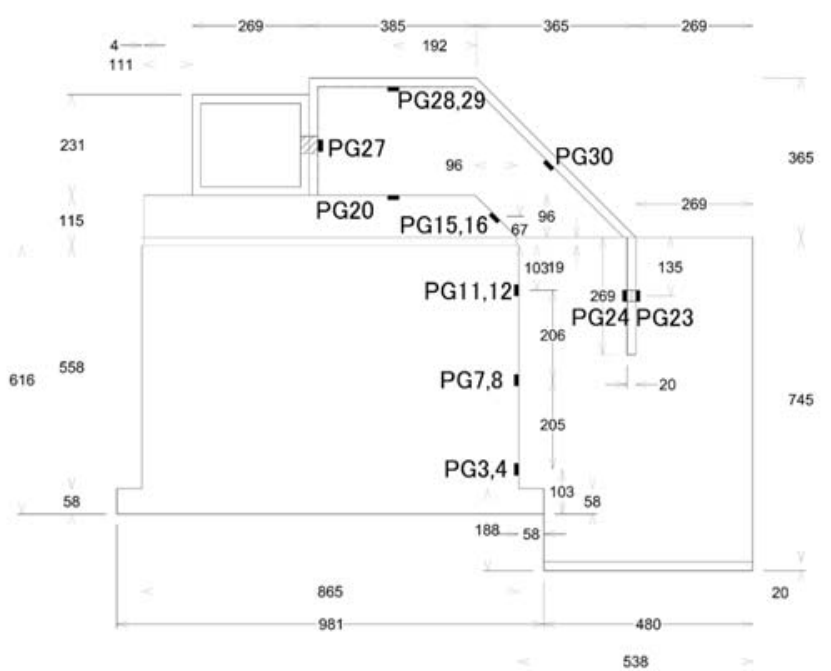

Figure 5. Layout of pressure transducers.

All of the experiments were conducted in regular waves and under the wave conditions indicated in Table 1. The area ratio of the air hole at the top of the air chamber and cross-section of the air chamber $\varepsilon$ was set up to 1/100 (Large Hole, LH) or 1/300 (Small Hole, SH).

Table 1. Experimental conditions of the medium-scale experiments.

\begin{tabular}{|c|c|c|c|c|c|}
\hline $\begin{array}{l}\text { Water } \\
\text { Level }\end{array}$ & $\begin{array}{l}\text { Air } \\
\text { hole }\end{array}$ & $\begin{array}{c}\text { Wave period } \\
T(\mathrm{~s})\end{array}$ & & $\begin{array}{l}\text { ive he } \\
H(\mathrm{~m})\end{array}$ & \\
\hline \multirow{12}{*}{$\begin{array}{l}\text { L.W.L. } \\
\pm 0.0\end{array}$} & \multirow{6}{*}{$\begin{array}{c}\text { LH } \\
(1 / 100)\end{array}$} & 1.52 & 0.05 & 0.10 & 0.15 \\
\hline & & 2.03 & 0.05 & 0.10 & 0.15 \\
\hline & & 2.53 & 0.05 & 0.10 & 0.15 \\
\hline & & 3.04 & 0.05 & 0.10 & 0.15 \\
\hline & & 3.54 & 0.05 & 0.10 & 0.15 \\
\hline & & 4.05 & 0.05 & 0.10 & 0.15 \\
\hline & \multirow{6}{*}{$\begin{array}{c}\text { SH } \\
(1 / 300)\end{array}$} & 1.52 & 0.05 & 0.10 & 0.15 \\
\hline & & 2.03 & 0.05 & 0.10 & 0.15 \\
\hline & & 2.53 & 0.05 & 0.10 & 0.15 \\
\hline & & 3.04 & 0.05 & 0.10 & 0.15 \\
\hline & & 3.54 & 0.05 & 0.10 & 0.15 \\
\hline & & 4.05 & 0.05 & 0.10 & 0.15 \\
\hline
\end{tabular}

\section{Primary energy conversion efficiency}

Primary energy conversion efficiency $E^{(1)}$ is defined as the ratio of absorbed wave power $P_{A}$ and incident wave power $P_{I}$ as equation (1). $P_{A}$ can be calculated from the water level and the air pressure in the air chamber as equation (2).

$$
\begin{aligned}
& E^{(1)}=\frac{P_{A}}{P_{I}} \\
& P_{A}=\frac{A_{w}}{T} \int_{0}^{T} p(t) \frac{\partial \eta(t)}{\partial t} d t
\end{aligned}
$$

Where $A_{w}$ denotes the cross-sectional area of the water surface, $T$ is the wave period, $p$ is the air pressure in the chamber and $\eta$ is the water level. While $P_{I}$ is defined as follows:

$$
P_{I}=\frac{\rho g^{2} a^{2}}{8 \pi}\left(1+\frac{2 k h}{\sinh 2 k h}\right)(\tanh k h) T B
$$

Where $\rho$ denotes the density of the water, $g$ is the acceleration of gravity, $a$ is the amplitude of the incident wave, $k$ is the wave number, $h$ is the water depth and $B$ is the width of the converter. 
Figure 6 indicates $E^{(1)}$ of the PW-OWC converter with the air hole ratio of 1/100 and 1/300. $E^{(1)}$ becomes maximum when $h / L$ is $0.10-0.15$ in all cases, and the value in the case of $\varepsilon=1 / 100$ is generally larger than that of $\varepsilon=1 / 300$.

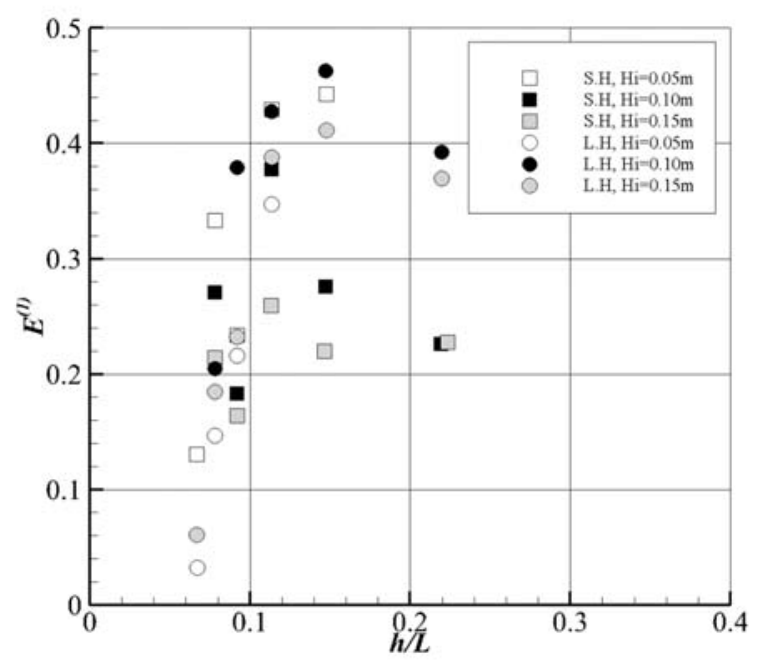

Figure 6. Primary energy conversion efficiency of PW-OWC converter $(\varepsilon=1 / 100$ and $\varepsilon=1 / 300)$.

Figure 7 compares $E^{(1)}$ of OWC type converter with and without a projecting wall. On average, the energy conversion efficiency of the PW-OWC is about $10 \%$ larger than that of the OWC without a projecting wall.

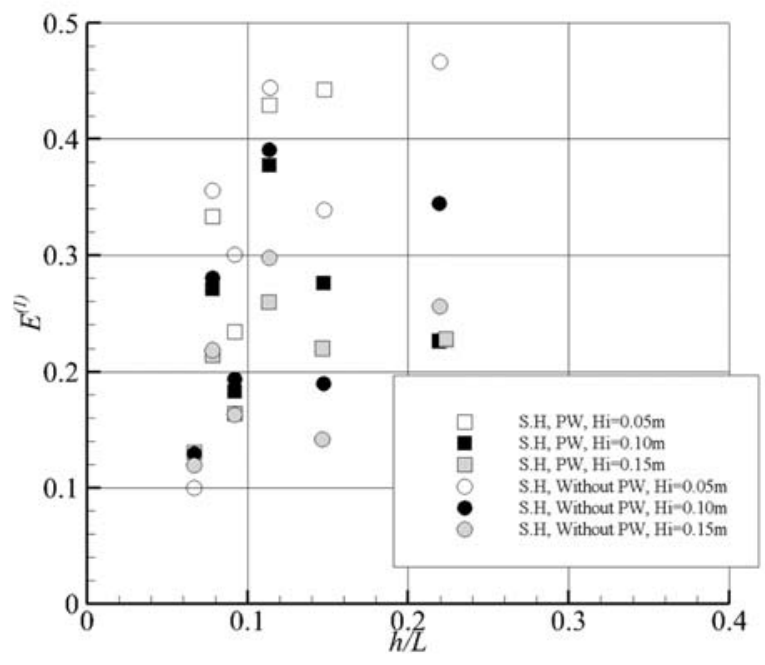

Figure 7. Primary energy conversion efficiency of OWC with and without projecting wall.

\section{LARGE-SCALE EXPERIMENTS}

\section{Experimental setup}

The large-scale model experiments were carried out in a large-wave flume (184 m long, $8 \mathrm{~m}$ high, $3.5 \mathrm{~m}$ wide) reproducing the same breakwater as the medium-scale experiments with a 1/6.7 scale model. The power generation efficiency of the impulse turbine, as well as the scale effect of primary energy conversion efficiency between medium and large scale experiments was examined. Figure 8 shows the cross section of the breakwater. The caisson was made of concrete, whereas the projecting wall and air chamber were made of steel plates. Figure 9 shows the caisson, projecting wall, air chamber, turbine and generator used in the large-scale experiments. 
Pressures acting on the caisson, projecting wall and in the air chamber were measured in the same way as in the medium-scale experiments. Figure 10 indicates the layout of pressure transducers. Wave gauges were also installed in the air chamber to measure the up-and-down motion of the water level, as well as in the front of the caisson to measure the wave height.

All the experiments were conducted in regular waves under the wave conditions indicated in Table 2. The area ratio of the air hole at the top of the air chamber and the cross-section of the air chamber $\varepsilon$ was set up to $1 / 100(\mathrm{LH})$ or $1 / 300(\mathrm{SH})$.

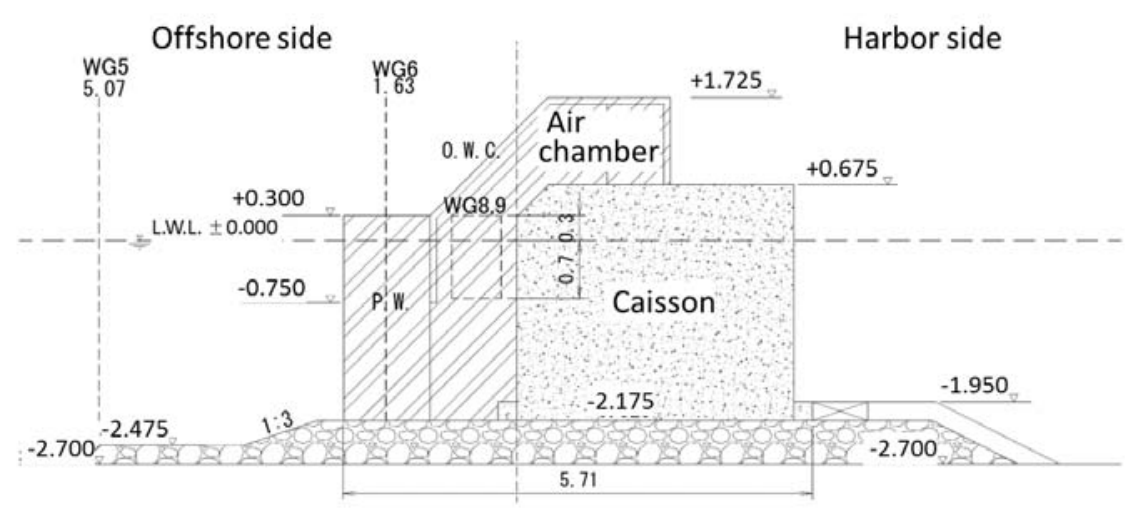

Figure 8. Cross-section of the wave power extracting breakwater in the large-scale experiments.

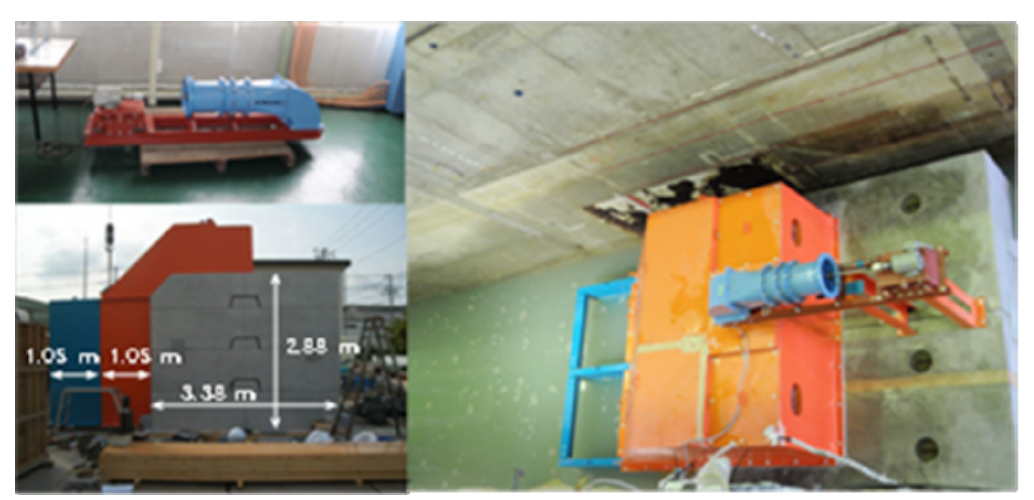

Figure 9. Model used in the large-scale experiments.

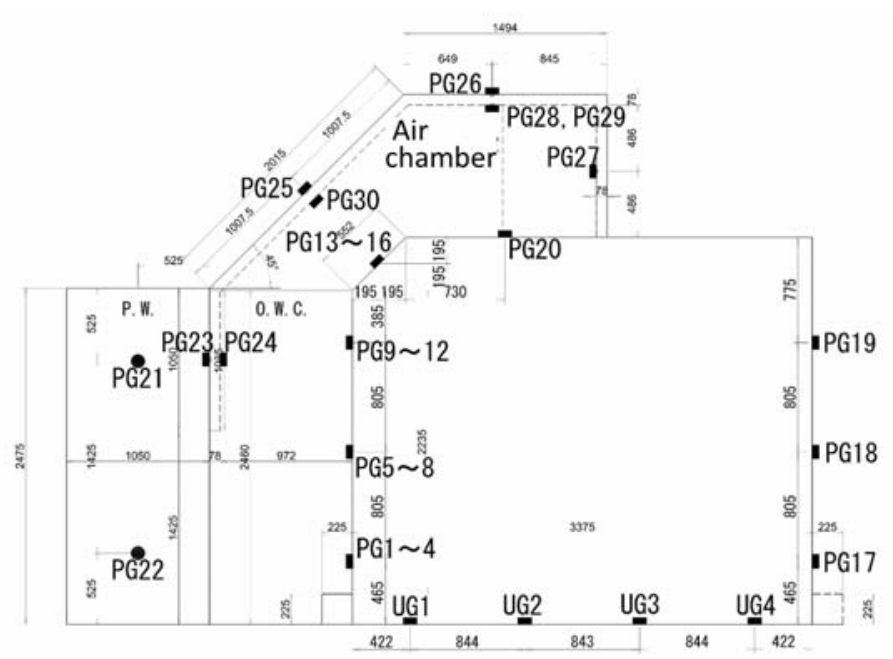

Figure 10. Layout of pressure transducers. 
Table 2. Layout of pressure transducers.

\begin{tabular}{|c|c|c|c|c|c|}
\hline $\begin{array}{l}\text { Water } \\
\text { Level }\end{array}$ & Air hole & $\begin{array}{c}\text { Wave period } \\
T(\mathrm{~s})\end{array}$ & \multicolumn{3}{|c|}{$\begin{array}{c}\text { Wave height } \\
H(\mathrm{~m})\end{array}$} \\
\hline \multirow{7}{*}{$\begin{array}{l}\text { L.W.L. } \\
\pm 0.0\end{array}$} & \multirow{4}{*}{$\mathrm{LH}(1 / 100)$} & 3.0 & 0.2 & 0.4 & 0.6 \\
\hline & & 4.0 & 0.2 & 0.4 & 0.6 \\
\hline & & 5.0 & 0.2 & 0.4 & 0.6 \\
\hline & & 6.0 & 0.2 & 0.4 & 0.6 \\
\hline & \multirow{3}{*}{$\begin{array}{c}\mathrm{SH}(1 / 300) \\
\text { or } \\
\text { Turbine }\end{array}$} & 3.0 & 0.2 & 0.4 & 0.6 \\
\hline & & 4.0 & 0.2 & 0.4 & 0.6 \\
\hline & & 5.0 & 0.2 & 0.4 & 0.6 \\
\hline
\end{tabular}

\section{Primary energy conversion efficiency}

Figure 11 presents an example of the time series of the wave profile in the air chamber, the average pressure, and the absorbed wave power obtained from equation (2). The phases of the average pressure and the absorbed wave power are almost the same, whereas that of the wave profile is delayed by 90 degrees.

Figure 12 compares the primary energy conversion efficiency $E^{(1)}$ of the PW-OWC converter with the air hole ratio of $1 / 100$ and $1 / 300$. The value of $E^{(1)}$ is generally larger in the case of $\varepsilon=1 / 100$ than that of $\varepsilon=1 / 300$; that is, the results are the same as in the medium-scale experiments. $E^{(1)}$ with the impulse turbine is also indicated in the figure, and shows the same tendency as of $\varepsilon=1 / 300$.

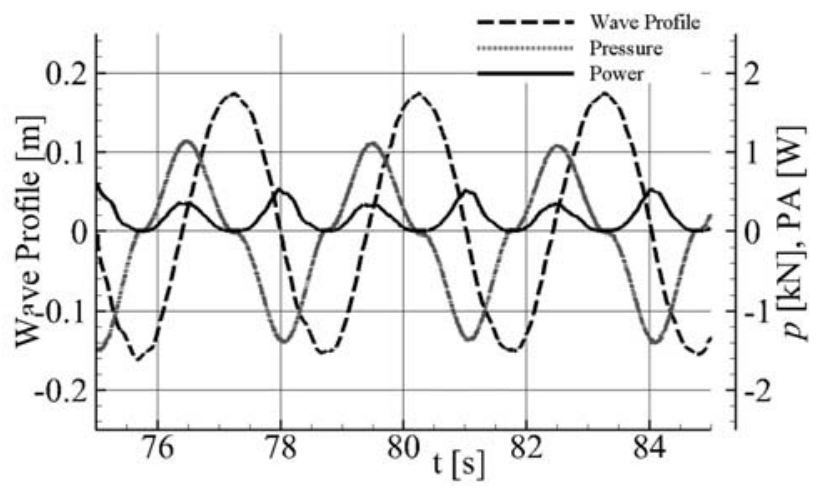

Figure 11. Time series of wave profile, average pressure and absorbed wave power.

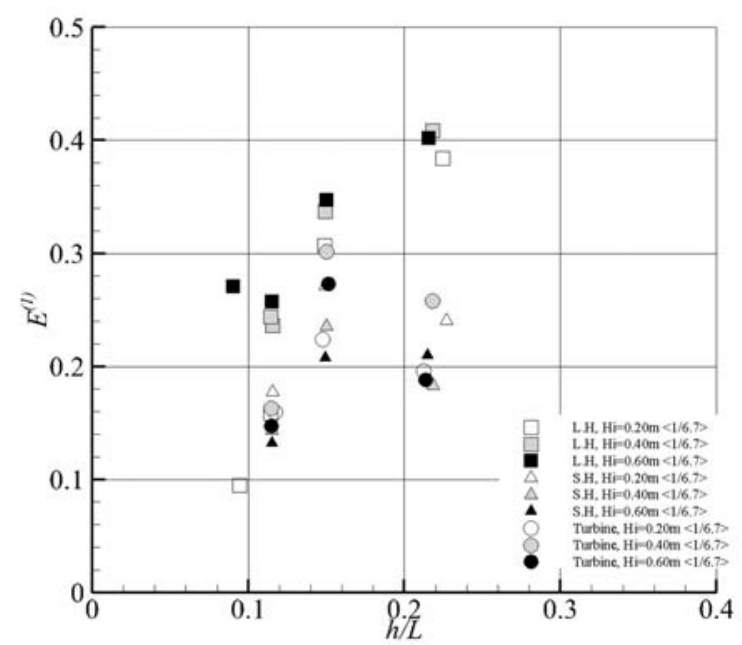

Figure 12. Primary energy conversion efficiency of the PW-OWC converter ( $\varepsilon=1 / 100, \varepsilon=1 / 300$ and turbine). 
Figure 13 compares $E^{(1)}$ in the medium-scale and large-scale experiments. The value of the largescale experiments is about $20-30 \%$ smaller than that of the medium-scale experiments. This effect can be estimated by the calculation method used by Ojima et al.

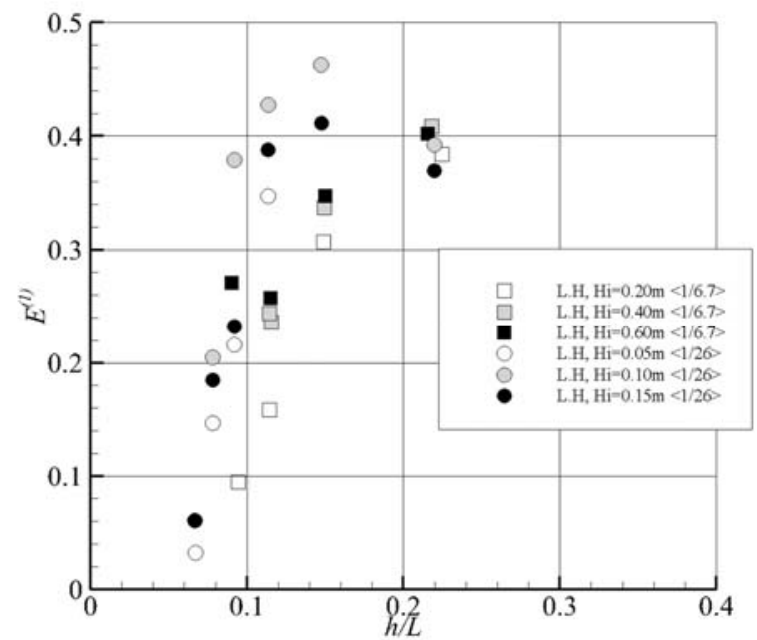

Figure 13. Primary energy conversion efficiency in the medium-scale and large scale-experiments $(\varepsilon=1 / 100)$.

\section{Turbine output power}

Figure 14 shows the time series of revolution $n$, torque $T$ and output power $P$ of the turbine in the large scale experiments. Output power $P$ was obtained by equation (4).

$$
P(\mathrm{w})=n(\mathrm{r} / \mathrm{min}) * T(\mathrm{~N} \cdot \mathrm{m}) / 9.55
$$

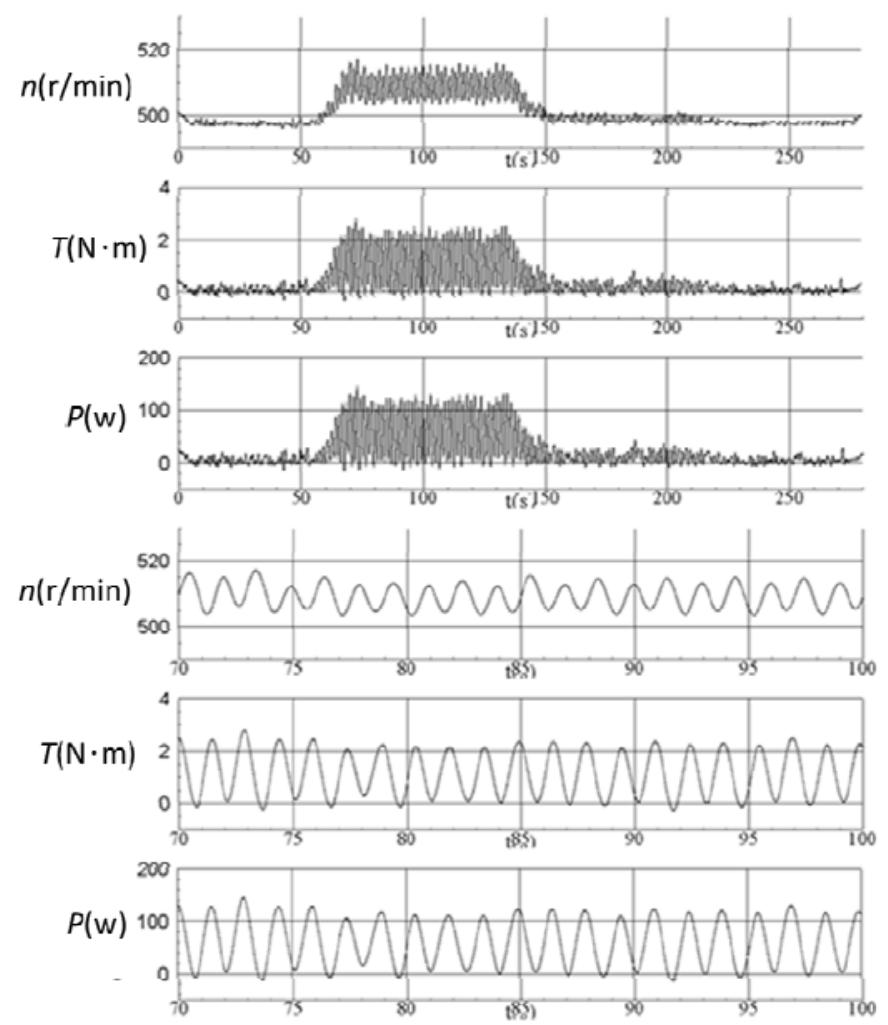

Figure 14. Revolution, torque and output power of the turbine. 
Figure 15 indicates the secondary energy conversion efficiency $E^{(2)}$ defined by $P / P_{A} . E^{(2)}$ ranges from 0.20 to 0.42 and becomes small when the wave period is short and the wave height is large. This is because the turbine could not follow the increase in the speed of the motion of the water surface in the air chamber. The performance characteristics of the turbine must be improved in order to increase $E^{(2)}$.

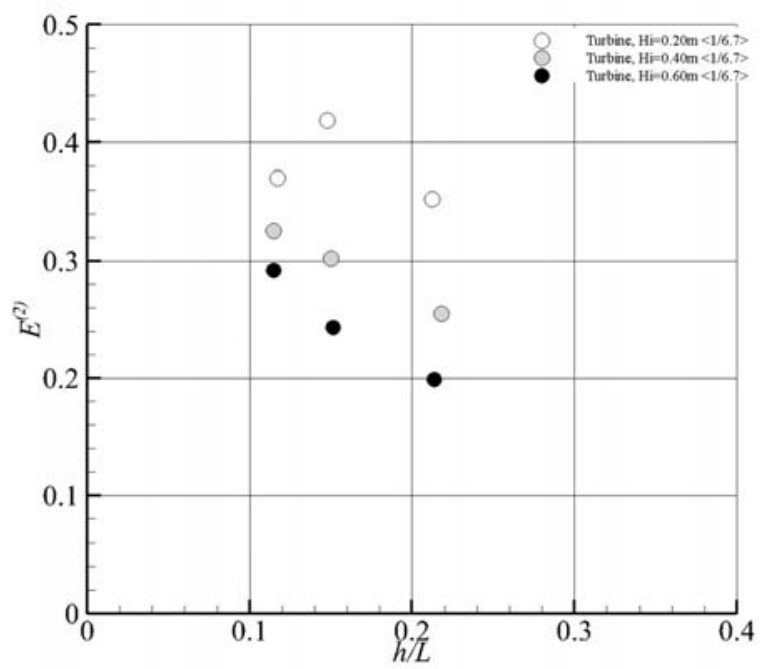

Figure 15. Secondary energy conversion efficiency.

\section{FIELD TEST}

\section{Experimental setup}

Prototype field test was conducted in Sakata Port, Yamagata Prefecture, northern part of Japan. Sakata port has a line of unique wave dissipating seawalls of up-right type (Fig. 16). This seawall has two chambers inside, a fore-chamber and a back-chamber, and they are connected by the openings. Incident waves can go into these chambers freely, and top of each chambers are open to the air (Fig. 17).

Figure 18 shows the experimental facility installed on the existing seawall. We use the forechamber as PW, and the back-chamber as OWC. Both chambers are same-size (W3.5m $\times$ L3.5m $\times \mathrm{H} 5.5 \mathrm{~m})$. Incident wave is usual small except winter, and the design wave height $H_{1 / 3}$ and wave period $T_{1 / 3}$ is $6.0 \mathrm{~m}$ and $14 \mathrm{~s}$, respectively.

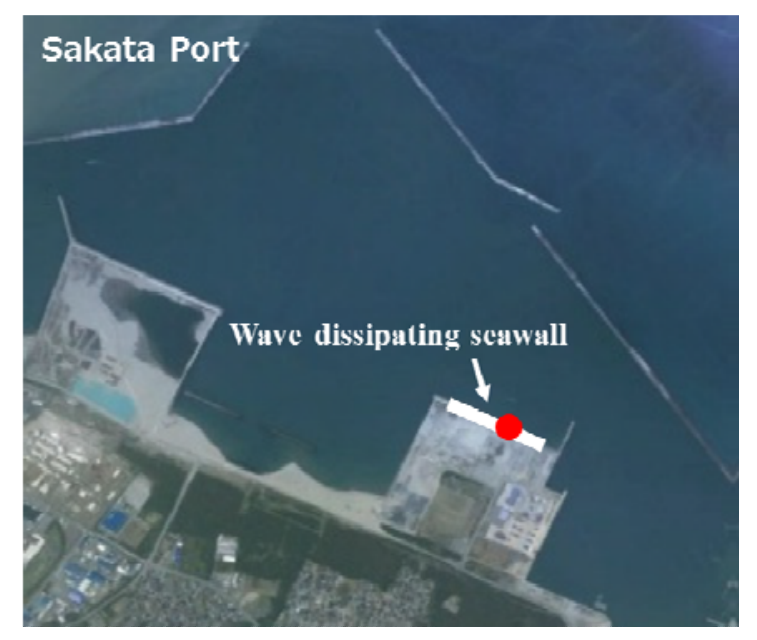

Figure 16. Location of wave dissipating seawall in Sakata Port. 


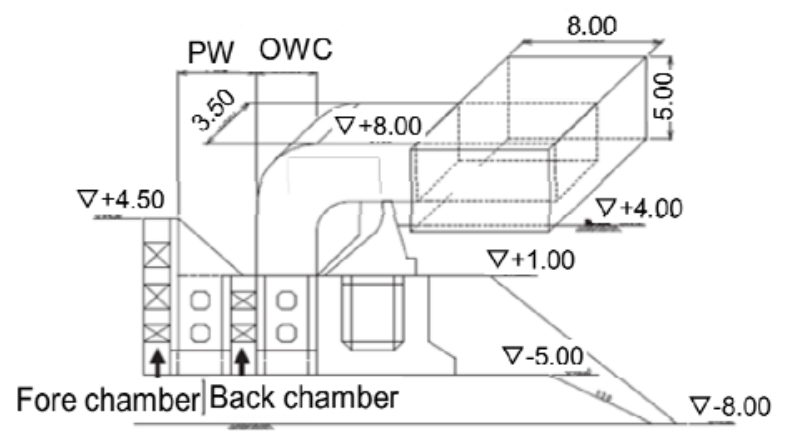

Figure 17. Cross section of wave dissipating seawall and PW-OWC.

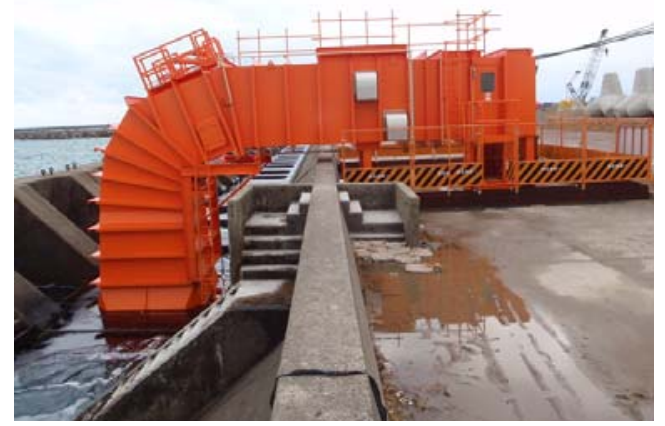

Figure 18. Wave power generation facility in Sakata Port.

\section{Air pressure and generation properties}

An example of the scatter diagrams of air chamber pressure and the turbine torque are presented in Fig.19, observed on February 13, 2015. Observed wave height $H_{1 / 3}$ and wave period $T_{1 / 3}$ is $1.9 \mathrm{~m}$ and $7.1 \mathrm{~s}$, respectively at OWC. The right side of each figure expresses a positive pressure (aspirating phase), and the left side negative pressure (inspiriting phase). The platted dots in the figure are asymmetric, and the turbine torque as well as generated electricity shows larger efficiency when the air flow is aspiring.

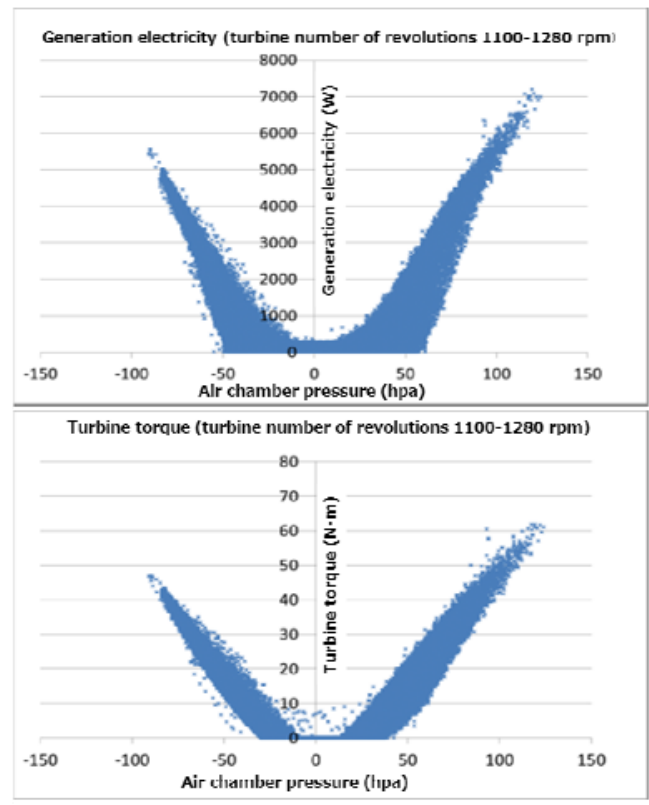

Figure 19. Air chamber pressure and generation properties. 


\section{CONCLUSIONS}

The medium-scale (1/26) and large-scale (1/6.7) hydraulic model experiments on a new oscillating water column with projecting wall (PW-OWC) wave power extracting breakwater were carried out in order to investigate the energy conversion efficiency of the projecting wall. The primary energy conversion efficiency of the PW-OWC converter is about $10 \%$ larger than that of the standard OWC in medium-scale experiments. The primary energy conversion efficiency in the large-scale experiments is about $20-30 \%$ smaller than that of the medium-scale experiments. The secondary energy conversion efficiency ranges from 0.20 to 0.42 and becomes small when the wave period is short and the wave height is large. Prototype field test was also conducted and the turbine torque and generated electricity as well as incident waves are obtained.

\section{ACKNOWLEDGMENT}

This work is part of an ongoing project of "Research and Development of Natural Energy Technologies Based on Wind Power, Etc.” supported by New Energy and industrial Technology Development Organization (NEDO).

\section{REFERENCES}

Takahashi, S., Nakada, H., Ohneda, H., and Shikamori, M. 1992. Wave Power Conversion by a Prototype Wave Power Extracting Caisson in Sakata Port, Proceedings of 23rd International Conference on Coastal Engineering, ASCE, 3440-3453.

Ikoma, T., Masuda, K., Omori, H., Osawa, H., Miyazaki, T., and Kihara, K. 2013. Research on the effect of the primary energy conversion efficiency by projecting wall of OWC-type wave energy converter, Journal of JSCE, Ser.B3 (Ocean Engineering), Vol.69, No.2, 120-125. (in Japanese).

Ojima, R., Goda, Y., and Suzumura, S. 1983. Analysis of Efficiency of Pneumatic-type Wave Power Extractors Utilizing Caisson Breakwaters, Report of the Port and Harbour Research Institute, Vol.22, No.3, 125-158. (in Japanese). 